Commun. Korean Math. Soc. 28 (2013), No. 3, pp. 463-480

http://dx.doi.org/10.4134/CKMS.2013.28.3.463

\title{
ON NIL GENERALIZED POWER SERIESWISE ARMENDARIZ RINGS
}

\author{
OUYANG LUNQUn AND LiU JinWANG
}

\begin{abstract}
We in this note introduce a concept, so called nil generalized power serieswise Armendariz ring, that is a generalization of both $S$-Armendariz rings and nil power serieswise Armendariz rings. We first observe the basic properties of nil generalized power serieswise Armendariz rings, constructing typical examples. We next study the relationship between the nilpotent property of $R$ and that of the generalized power series ring $\left[\left[R^{S, \leq}\right]\right]$ whenever $R$ is nil generalized power serieswise Armendariz.
\end{abstract}

\section{Introduction}

Throughout this paper $R$ denotes an associative ring with identity and $\operatorname{nil}(R)$ stands for the set of all nilpotent elements of $R$. A ring $R$ is called an $N I$ ring if $\operatorname{nil}(R)$ forms an ideal, and a ring $R$ is said to be semicommutative if for all $a, b \in R, a b=0$ implies $a R b=0$. Let $I$ be an ideal of $R, I$ is said to be semicommutative if $I$ is considered as a semicommutative ring without identity, and $I$ is said to be nilpotent if $I^{n}=0$ for some positive integer $n$. Let $U$ be a subset of $R$. We denote by $U[[x]]$ the set $\left\{f(x)=\sum_{i=0}^{\infty} a_{i} x^{i} \in R[[x]] \mid a_{i} \in U, i=0,1, \ldots\right\}$.

A ring $R$ is called Armendariz if whenever polynomials $f(x)=\sum_{i=0}^{m} a_{i} x^{i}$, $g(x)=\sum_{j=0}^{n} b_{j} x^{j} \in R[x]$ satisfy $f(x) g(x)=0$, then $a_{i} b_{j}=0$ for each $i, j$. The term Armendariz was introduced by Rege and Chhawchharia [13]. This nomenclature was used by them since it was Armendariz [3, Lemma 1] who initially showed that a reduced ring (i.e., a ring without nonzero nilpotent elements) always satisfies this condition. Armendariz rings are thus a generalization of reduced rings, and therefore, nilpotent elements play an important role in this class of rings. There are many examples of rings with nilpotent elements which are Armendariz. In fact, in [1], Anderson and Camillo proved that if $n>2$, then $R[x] /\left(x^{n}\right)$ is an Armendariz ring if and only if $R$ is reduced.

Received October 25, 2012.

2010 Mathematics Subject Classification. 16W60.

Key words and phrases. nil generalized power serieswise Armendariz, generalized power series ring, nilpotent property. 
N. K. Kim et al. [8] studied a generalization of Armendariz rings, which they called power serieswise Armendariz rings. A ring $R$ is called power serieswise Armendariz if whenever power series $f(x)=\sum_{i=0}^{\infty} a_{i} x^{i}, g(x)=\sum_{j=0}^{\infty} b_{j} x^{j} \in$ $R[[x]]$ satisfy $f(x) g(x)=0$, then $a_{i} b_{j}=0$ for all $i, j$. As a generalization of power serieswise Armendariz rings, S. Hizem in [7] introduced the concept of nil power serieswise Armendariz rings and Z. K. Liu in [10] introduced the notion of $S$-Armendariz rings, respectively. Following S. Hizem [7], a ring $R$ is called nil power serieswise Armendariz if whenever power series $f(x)=\sum_{i=0}^{\infty} a_{i} x^{i}$, $g(x)=\sum_{j=0}^{\infty} b_{j} x^{j} \in R[[x]]$ satisfy $f(x) g(x) \in \operatorname{nil}(R)[[x]]$, then $a_{i} b_{j} \in \operatorname{nil}(R)$ for all $i, j$. Let $(S, \leq)$ be a cancellative torsion-free strictly ordered monoid and let $\left[\left[R^{S,} \leq\right]\right]$ be a generalized power series ring over $R$. According to Z. K. Liu [10], the ring $R$ is called $S$-Armendariz if whenever $f, g \in\left[\left[R^{S, \leq}\right]\right]$ satisfy $f g=0$, then $f(u) g(v)=0$ for each $u, v \in S$.

In this paper we investigate a generalization of both nil power serieswise Armendariz rings and $S$-Armendariz rings which we call nil generalized power serieswise Armendariz rings. We first observe the basic properties of nil generalized power serieswise Armendariz rings, constructing typical examples. We next study the relationship between the nilpotent property of $R$ and that of the generalized power series ring $\left[\left[R^{S, \leq}\right]\right]$ whenever $R$ is nil generalized power serieswise Armendariz.

Now let us briefly review the concept of generalized power series rings. Let $(S, \leq)$ be an ordered set. Recall that $(S, \leq)$ is artinian if every strictly decreasing sequence of elements of $S$ is finite, and that $(S, \leq)$ is narrow if every subset of pairwise order-incomparable elements of $S$ is finite. Let $S$ be a commutative monoid. Unless stated otherwise, the operation of $S$ shall be denoted additively, the neutral element by 0 and $|S| \geq 2$.

Let $(S, \leq)$ be a strictly ordered monoid (that is, $(S, \leq)$ is an ordered monoid satisfying the condition that, if $s, s^{\prime}, t \in S$ and $s<s^{\prime}$, then $\left.s+t<s^{\prime}+t\right)$ and $R$ a ring. Let $\left[\left[R^{S, \leq}\right]\right]$ be the set of all maps $f: S \longrightarrow R$ such that $\operatorname{supp}(f)=\{s \in S \mid f(s) \neq 0\}$ is artinian and narrow. With pointwise addition, $\left[\left[R^{S, \leq}\right]\right]$ is an abelian additive group. For every $s \in S$ and $f, g \in\left[\left[R^{S, \leq}\right]\right]$, let $X_{s}(f, g)=\{(u, v) \in S \times S \mid s=u+v, f(u) \neq 0, g(v) \neq 0\}$. It follows from [15, Section 4.1] that $X_{s}(f, g)$ is finite. This fact allows to define the operation of convolution:

$$
(f g)(s)=\sum_{(u, v) \in X_{s}(f, g)} f(u) g(v) .
$$

With this operation of convolution, and pointwise addition, $\left[\left[R^{S, \leq}\right]\right]$ becomes a ring (see $[14,15,16]$ ), which is called the ring of generalized power series. The elements of $\left[\left[R^{S, \leq}\right]\right]$ are called generalized power series with coefficients in $R$ and exponents in $S$.

Let $s \in S, r \in R$. We define $C_{r}^{s} \in\left[\left[R^{S,} \leq\right]\right]$ as follows:

$$
C_{r}^{s}(s)=r, \quad C_{r}^{s}(t)=0 \quad(s \neq t \in S) .
$$


It is clear that $r \rightarrow C_{r}^{0}$ is a ring embedding of $R$ into $\left[\left[R^{S, \leq}\right]\right]$. So we can regard $R$ as a subring of $\left[\left[R^{S, \leq}\right]\right]$, and for any $f \in\left[\left[R^{S, \leq}\right]\right], r \in R, f r=f C_{r}^{0}$.

Given a subset $U \subseteq R,\left[\left[U^{S,} \leq\right]\right]$ means the set $\left\{f \in\left[\left[R^{S, \leq}\right]\right] \mid f(s) \in U, s \in\right.$ $\operatorname{supp}(f)\}$. In particular, $\left[\left[\operatorname{nil}(R)^{S, \leq}\right]\right]$ stands for the set $\left\{f \in\left[\left[R^{S, \leq}\right]\right] \mid f(s) \in\right.$ $\operatorname{nil}(R), s \in \operatorname{supp}(f)\}$. For any $s \in S$ and any nature number $n$, we denote by $n s$ the sum of $\mathrm{n}$ copies of $\mathrm{s}$. Other concepts and notations not defined here can be found in $[14,15,16]$.

\section{Nil generalized power serieswise Armendariz rings}

In this section, we first give the following concept, so called nil generalized power serieswise Armendariz ring, that is both a generalization of $S$ Armendariz rings and nil power serieswise Armendariz rings.

Definition 2.1. Let $(S, \leq)$ be a strictly ordered monoid. A ring $R$ is called nil generalized power serieswise Armendariz if whenever $f, g \in\left[\left[R^{S, \leq}\right]\right]$ satisfy $f g \in\left[\left[\operatorname{nil}(R)^{S, \leq}\right]\right]$, then $f(u) g(v) \in \operatorname{nil}(R)$ for each $u, v \in S$.

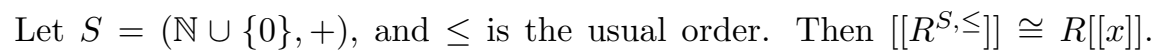
So the ring $R$ is nil generalized power serieswise Armendariz if and only if $R$ is nil power serieswise Armendariz. Hence a nil generalized power serieswise Armendariz ring is a generalization of a nil power serieswise Armendariz ring. Obviously, any subring of a nil generalized power serieswise Armendariz ring is also nil generalized power serieswise Armendariz.

The following proposition enable us to generate more examples of nil generalized power serieswise Armendariz rings.

Proposition 2.2. Let $S$ be a torsion-free and cancellative monoid, $\leq$ a strict order on $S$, and $R$ a ring. Then the following conditions are equivalent:

(1) $R$ is nil generalized power serieswise Armendariz.

(2) $R$ is an NI ring.

Proof. (1) $\Rightarrow(2)$ Suppose that $a \in \operatorname{nil}(R), r \in R$, and $0 \neq s \in S$. Define $f \in\left[\left[R^{S, \leq}\right]\right]$ via

$$
f(x)=\left\{\begin{aligned}
1 & \text { if } \quad x=0 \\
r^{n} & \text { if } \quad x=n s, n=1,2, \ldots, \\
0 & \text { otherwise. }
\end{aligned}\right.
$$

Then $C_{a}^{0}\left(C_{1}^{0}-C_{r}^{s}\right) f=C_{a}^{0} \in\left[\left[\operatorname{nil}(R)^{S, \leq}\right]\right]$. Hence $\left(C_{a}^{0}\left(C_{1}^{0}-C_{r}^{s}\right)\right)(0) f(s)=a r \in$ $\operatorname{nil}(R)$ because $R$ is nil generalized power serieswise Armendariz. Note that ar $\in \operatorname{nil}(R)$ implies $r a \in \operatorname{nil}(R)$.

Now we show that $x+y z \in \operatorname{nil}(R)$ for all $x, y, z \in \operatorname{nil}(R)$. Since $y \in \operatorname{nil}(R)$, we have $-y(x+y z) \in \operatorname{nil}(R)$. Let $0 \neq s \in S$. Construct $h, g \in\left[\left[R^{S, \leq}\right]\right]$ as follows:

$$
h(x)=\left\{\begin{array}{ll}
1 & \text { if } x=0, \\
-y & \text { if } x=s, \\
0 & \text { otherwise }
\end{array} \quad \text { and } \quad g(x)= \begin{cases}z & \text { if } x=0 \\
x+y z & \text { if } x=s \\
0 & \text { otherwise }\end{cases}\right.
$$


Then $h g \in\left[\left[\operatorname{nil}(R)^{S, \leq}\right]\right]$. Since $R$ is nil generalized power serieswise Armendariz, $h(0) g(s)=x+y z \in \operatorname{nil}(R)$. Then by analogy with the proof of $\mathrm{R}$. Antoine [2], Lemma 3.1(d), we can show that $n i l(R)$ is an ideal, and so $R$ is an $N I$ ring.

$(2) \Rightarrow(1)$ Assume that $R$ is an $N I$ ring, and $f, g \in\left[\left[R^{S, \leq}\right]\right]$ are such that $f g \in\left[\left[\operatorname{nil}(R)^{S, \leq}\right]\right]$. Then $\bar{f} \bar{g}=\overline{0}$, where $\bar{f}, \bar{g}$ are the corresponding generalized power series of $f, g$ in $\left[\left[(R / \operatorname{nil}(R))^{S, \leq}\right]\right]$. Observe that $R / \operatorname{nil}(R)$ is reduced and hence $S$-Armendariz by [10]. Thus $\bar{f}(u) \bar{g}(v)=\overline{0}$ for any $u, v \in S$. Hence $f(u) g(v) \in \operatorname{nil}(R)$ for any $u, v \in S$. Therefore $R$ is nil generalized power serieswise Armendariz.

Corollary 2.3. Let $\left(S_{1}, \leq_{1}\right),\left(S_{2}, \leq_{2}\right), \ldots,\left(S_{n}, \leq_{n}\right)$ be cancellative torsion-free strictly ordered monoids. Denote by $($ lex $\leq)$ and (revlex $\leq)$ the lexicographic order, the reverse lexicographic order, respectively, on the monoid $S_{1} \times S_{2} \times$ $\cdots \times S_{n}$. Then the following conditions are equivalent:

(1) $R$ is an $N I$ ring.

(2) $R$ is nil generalized power serieswise Armendariz for any ordered monoid $\left(S_{i}, \leq_{i}\right)$

(3) $R$ is nil generalized power serieswise Armendariz for ordered monoid $\left(S_{1} \times S_{2} \times \cdots \times S_{n},(\right.$ lex $\left.\leq)\right)$.

(4) $R$ is nil generalized power serieswise Armendariz for ordered monoid $\left(S_{1} \times S_{2} \times \cdots \times S_{n},(\right.$ revlex $\left.\leq)\right)$.

Proof. It is easy to see that $\left(S_{1} \times S_{2} \times \cdots \times S_{n},(\right.$ lex $\left.\leq)\right)$ and $\left(S_{1} \times S_{2} \times \cdots \times\right.$ $S_{n},($ revlex $\left.\leq)\right)$ are cancellative torsion-free strictly ordered monoids. Therefore we complete the proofs of $(1) \Leftrightarrow(2),(1) \Leftrightarrow(3)$ and $(1) \Leftrightarrow(4)$ by Proposition 2.2 .

A ring $R$ is called $n$ nil power serieswise Armendariz if $f=\sum a_{i_{1}, i_{2}, \ldots, i_{n}} x_{1}^{i_{1}} x_{2}^{i_{2}}$ $\cdots x_{n}^{i_{n}}, g=\sum b_{j_{1}, j_{2}, \ldots, j_{n}} x_{1}^{j_{1}} x_{2}^{j_{2}} \cdots x_{n}^{j_{n}} \in R\left[\left[x_{1}, x_{2}, \ldots, x_{n}\right]\right]$ satisfy $f g \in \operatorname{nil}(R)$ $\left[\left[x_{1}, x_{2}, \ldots, x_{n}\right]\right]$, then $a_{i_{1}, i_{2}, \ldots, i_{n}} b_{j_{1}, j_{2}, \ldots, j_{n}} \in \operatorname{nil}(R)$ for all $i_{1}, i_{2}, \ldots, i_{n}$ and $j_{1}, j_{2}, \ldots, j_{n}$.

Corollary 2.4. Let $R$ be a ring. Then the following conditions are equivalent.

(1) The ring $R$ is an $N I$ ring.

(2) The ring $R$ is nil power serieswise Armendariz.

(3) The ring $R$ is $n$ nil power serieswise Armendariz.

Proof. (1) $\Leftrightarrow(2)$ is ciear.

$(1) \Leftrightarrow(3)$ Note that if $S=\mathbb{N}^{n}$, with the product order, or the lexicographic order, or the reverse lexicographic order, then $\left[\left[R^{S, \leq}\right]\right] \cong R\left[\left[x_{1}, x_{2}, \ldots, x_{n}\right]\right]$ (see $[15$, Example 4]). Then by Corollary 2.3, we complete the proof.

Let $R$ be a ring and let 


$$
\begin{aligned}
& T_{n}(R)=\left(\left\langle\begin{array}{cccc}
a_{11} & a_{12} & \cdots & a_{1 n} \\
0 & a_{22} & \cdots & a_{2 n} \\
\vdots & \vdots & \ddots & \vdots \\
0 & 0 & \cdots & a_{n n}
\end{array}\right) \mid a_{i j} \in R\right\}, \\
& S_{n}(R)=\left(\left(\begin{array}{cccc}
a & a_{12} & \cdots & a_{1 n} \\
0 & a & \cdots & a_{2 n} \\
\vdots & \vdots & \ddots & \vdots \\
0 & 0 & \cdots & a
\end{array}\right) \mid a, a_{i j} \in R\right\}, \\
& T(R, n)=\left\{\left(\begin{array}{cccc}
a_{1} & a_{2} & \cdots & a_{n} \\
0 & a_{1} & \cdots & a_{n-1} \\
\vdots & \vdots & \ddots & \vdots \\
0 & 0 & \cdots & a_{1}
\end{array}\right) \mid a_{i} \in R\right\} \\
& W(R)=\left\{\left(\begin{array}{lll}
a_{11} & 0 & 0 \\
a_{12} & a_{22} & a_{23} \\
0 & 0 & a_{33}
\end{array}\right) \mid a_{i j} \in R\right\}
\end{aligned}
$$

and $T(R, R)$ be the trivial extension of $R$ by $R$. They are all rings under usual matrix operations. Then we have the following results.

Proposition 2.5. Let $S$ be a torsion-free and cancellative monoid, $\leq$ a strict order on $S$. Then the following conditions are equivalent:

(1) $R$ is nil generalized power serieswise Armendariz.

(2) $T_{n}(R)$ is nil generalized power serieswise Armendariz.

(3) $S_{n}(R)$ is nil generalized power serieswise Armendariz.

(4) $T(R, n)$ is nil generalized power serieswise Armendariz.

(5) $W(R)$ is nil generalized power serieswise Armendariz.

(6) $T(R, R)$ is nil generalized power serieswise Armendariz.

(7) $R[x] /\left(x^{n}\right)$ is nil generalized power serieswise Armendariz for any $n \geq 2$.

Proof. (1) $\Rightarrow(2)$ Suppose that $R$ is nil generalized power serieswise Armendariz. Then by Proposition $2.2, R$ is an NI ring. Since

$$
\operatorname{nil}\left(T_{n}(R)\right)=\left(\begin{array}{cccc}
\operatorname{nil}(R) & R & \cdots & R \\
0 & \operatorname{nil}(R) & \cdots & R \\
\vdots & \vdots & \ddots & \vdots \\
0 & 0 & \cdots & \operatorname{nil}(R)
\end{array}\right)
$$

it is easy to see that $T_{n}(R)$ is an $N I$ ring. Then by Proposition $2.2, T_{n}(R)$ is nil generalized power serieswise Armendariz.

$(2) \Rightarrow(1)$ Note that any subring of a nil generalized power serieswise Armendariz ring is also nil generalized power serieswise Armendariz. Hence $(2) \Rightarrow(1)$ is straightforward.

Similarly, we can show that $(1) \Leftrightarrow(3),(1) \Leftrightarrow(4),(1) \Leftrightarrow(5)$, and (1) $\Leftrightarrow(6)$. 
The proof of $(1) \Leftrightarrow(7)$ follows from the fact that $R[x] /\left(x^{n}\right) \cong T(R, n)$ for any $n \geq 2$.

Let $M$ be an $R$ - $R$-bimodule. A $\mathbb{Z}$-bilinear map $\alpha: R \times R \longrightarrow M$ is called a Hochschild 2-cocycle if for all $\lambda_{1}, \lambda_{2}, \lambda_{3} \in R$, the following equation holds true:

$$
\alpha\left(\lambda_{1} \lambda_{2}, \lambda_{3}\right)-\alpha\left(\lambda_{1}, \lambda_{2} \lambda_{3}\right)=\lambda_{1} \alpha\left(\lambda_{2}, \lambda_{3}\right)-\alpha\left(\lambda_{1}, \lambda_{2}\right) \lambda_{3} .
$$

Given a Hochschild 2-cocycle $\alpha$, there is a ring $H_{\alpha}(R, M)$, called the Hochschild extension of $R$ by $M$ via $\alpha$, which is $R \oplus M$ as an abelian group, and the multiplication is defined by

$$
\left(r_{1}, m_{1}\right)\left(r_{2}, m_{2}\right)=\left(r_{1} r_{2}, r_{1} m_{2}+m_{1} r_{2}+\alpha\left(r_{1}, r_{2}\right)\right)
$$

for all $r_{1}, r_{2} \in R$ and all $m_{1}, m_{2} \in M$.

This is an associative ring [5]. If $\alpha=0$, the extension $\operatorname{ring} H_{0}(R, M)$ is the trivial extension of $R$ by $M$ in the literature. Note that the nilpotent elements of $H_{\alpha}(R, M)$ is $(\operatorname{nil}(R), M)$. Then we have the following result:

Proposition 2.6. Let $S$ be a torsion-free and cancellative monoid, $\leq$ a strict order on $S$. Then the following conditions are equivalent:

(1) $R$ is nil generalized power serieswise Armendariz.

(2) $H_{\alpha}(R, M)$ is nil generalized power serieswise Armendariz

Remark 2.7. A ring is called symmetric if $a b c=0$ implies $a c b=0$ for all $a, b, c \in R$. A ring $R$ is called reversible if $a b=0$ implies $b a=0$ for all $a, b \in R$. Let $S$ be a torsion-free and cancellative monoid, $\leq$ a strict order on $S$. Since commutative rings, reduced rings, symmetric rings, reversible rings, semicommutative rings and 2-primal rings are NI rings, by Proposition 2.2, they are nil generalized power serieswise Armendariz rings. Hence nil generalized power serieswise Armendariz rings forms a large class of rings.

Let $S$ be a torsion-free and cancellative monoid, $\leq$ a strict order on $S$. Then for any ring $R$, the $n$ by $n$ matrix $\operatorname{ring} M_{n}(R)$ is never nil generalized power serieswise Armendariz. In fact, consider $x=E_{12}$ and $y=-E_{21}$, where $E_{i j}$ denote the $(i, j)$-matrix unit. Then $x, y \in \operatorname{nil}\left(M_{n}(R)\right)$, but $x-y \notin \operatorname{nil}\left(M_{n}(R)\right)$. Hence $M_{n}(R)$ is not an $N I$ ring.

The next lemma is known for $S$-Armendariz rings (see [10, Proposition 3.2]).

Lemma 2.8. Let $S$ be a torsion-free and cancellative monoid, $\leq$ a strict order on $S$ and $R$ an $S$-Armendariz ring. If $f_{1}, f_{2}, \ldots, f_{n} \in\left[\left[R^{S, \leq}\right]\right]$ are such that $f_{1} f_{2} \cdots f_{n}=0$, then $f_{1}\left(u_{1}\right) f_{2}\left(u_{2}\right) \cdots f_{n}\left(u_{n}\right)=0$ for all $u_{1}, u_{2}, \ldots, u_{n} \in S$.

The following result shows that our definition of a nil generalized power serieswise Armendariz ring is an extension of the Zhongkui Liu's [10] S-Armendariz ring for the more general setting.

Proposition 2.9. Let $S$ be a torsion-free and cancellative monoid, $\leq$ a strict order on $S$. Then all $S$-Armendariz rings are nil generalized power serieswise Armendariz. 
Proof. Suppose that $R$ is $S$-Armendariz. Let $a \in \operatorname{nil}(R), r \in R$ and $0 \neq s \in S$. Define $m \in\left[\left[R^{S, \leq}\right]\right]$ via

$$
m(x)= \begin{cases}1 & \text { if } \quad x=0 \\ r^{n} & \text { if } x=n s, \quad n=1,2, \ldots \\ 0 & \text { otherwise. }\end{cases}
$$

Then $C_{a}^{0}\left(C_{1}^{0}-C_{r}^{s}\right) m=C_{a}^{0}$. Suppose $a^{k}=0$ for some positive integer $k$. Then $\left(C_{a}^{0}\left(C_{1}^{0}-C_{r}^{s}\right) m\right)^{k}=\left(C_{a}^{0}\right)^{k}=0$. Then by Lemma 2.8, $\left(C_{a}^{0}(0)\left(C_{1}^{0}-\right.\right.$ $\left.\left.C_{r}^{s}\right)(0) m(s)\right)^{k}=(a r)^{k}=0$, and so ar $\in \operatorname{nil}(R), r a \in \operatorname{nil}(R)$.

Let $a, b, c \in \operatorname{nil}(R)$. Without loss of generality, we may assume that $a$, $b, c$ are all nonzero nilpotent elements. Let $0 \neq s \in S$. Now we claim that $a+b c \in \operatorname{nil}(R)$. Define $f, g \in\left[\left[R^{S, \leq}\right]\right]$ via

$$
f(x)=\left\{\begin{array}{ll}
1 & \text { if } x=s, \\
-b & \text { if } x=2 s, \\
0 & \text { otherwise, }
\end{array} \quad \text { and } \quad g(x)= \begin{cases}c & \text { if } x=s \\
a+b c & \text { if } x=2 s \\
0 & \text { otherwise }\end{cases}\right.
$$

Let $h=f g$. Then $\operatorname{supp}(h)=\operatorname{supp}(f g)=\{2 s, 3 s, 4 s\}, h(2 s)=c \in \operatorname{nil}(R)$, $h(3 s)=a \in \operatorname{nil}(R)$ and $h(4 s)=-b(a+b c) \in \operatorname{nil}(R)$. Let $k$ be a positive integer such that

$$
(h(2 s))^{k}=(h(3 s))^{k}=(h(4 s))^{k}=c^{k}=a^{k}=(-b(a+b c))^{k}=0 .
$$

Now we wish to claim that $(f g)^{3 k}=h^{3 k}=0$. For any $w \in S$,

$$
h^{3 k}(w)=\sum_{\left(u_{1}, \ldots, u_{3 k}\right) \in X_{w}(\underbrace{h, \ldots, h}_{3 k})} h\left(u_{1}\right) h\left(u_{2}\right) \cdots h\left(u_{3 k}\right),
$$

where $u_{i} \in\{2 s, 3 s, 4 s\}$ for all $1 \leq i \leq 3 k$. Consider each

$$
\begin{aligned}
& \left(u_{1}, \ldots, u_{3 k}\right) \in X_{w}(\underbrace{h, \ldots, h}_{3 k}) \\
= & \left\{\left(u_{1}, \ldots, u_{3 k}\right) \mid u_{1}+\cdots+u_{3 k}=w, u_{i} \in\{2 s, 3 s, 4 s\}, 1 \leq i \leq 3 k\right\} .
\end{aligned}
$$

It would contain at least $k, u_{j_{0}}$, where $u_{j_{0}} \in\{2 s, 3 s, 4 s\}$. Suppose that

$$
u_{r_{1}}=u_{r_{2}}=\cdots=u_{r_{k}}=u_{j_{0}}
$$

for some

$$
1 \leq r_{1}<r_{2}<\cdots<r_{k} \leq 3 k .
$$

For each $u_{v} \neq u_{r_{t}}, 1 \leq t \leq k$, define $h_{v}^{\prime} \in\left[\left[R^{S, \leq]}\right]\right.$ via

$$
h_{v}^{\prime}(x)= \begin{cases}1 & \text { if } x=0 \\ \left(h\left(u_{v}\right)\right)^{p} & \text { if } x=p u_{v}, p=1,2, \ldots, k-1 \\ 0 & \text { otherwise. }\end{cases}
$$


Then $\left(C_{1}^{0}-C_{h\left(u_{v}\right)}^{u_{v}}\right) h_{v}^{\prime}=C_{1}^{0}$, and $\left(C_{1}^{0}-C_{h\left(u_{v}\right)}^{u_{v}}\right)(0) h_{v}^{\prime}\left(u_{v}\right)=h\left(u_{v}\right)$. For convenience we write $h\left(u_{1}\right) h\left(u_{2}\right) \cdots h\left(u_{3 k}\right)$ as

$$
\begin{aligned}
& h\left(u_{1}\right) \cdots h\left(u_{r_{1}-1}\right) h\left(u_{j_{0}}\right) h\left(u_{r_{1}+1}\right) \cdots h\left(u_{r_{2}-1}\right) h\left(u_{j_{0}}\right) \\
& \cdots h\left(u_{r_{k}-1}\right) h\left(u_{j_{0}}\right) \cdots h\left(u_{3 k}\right) .
\end{aligned}
$$

By replacing each $h\left(u_{v}\right)\left(u_{v} \neq u_{r_{t}}, 1 \leq t \leq k\right)$ by the product $\left(C_{1}^{0}-\right.$ $\left.C_{h\left(u_{v}\right)}^{u_{v}}\right) h_{v}^{\prime}$, each $h\left(u_{j_{0}}\right)$ by $C_{h\left(u_{j_{0}}\right)}^{0}$, and consider the condition that $\left(h\left(u_{j_{0}}\right)\right)^{k}=$ 0 , we have

$$
\begin{aligned}
& \left(C_{1}^{0}-C_{h\left(u_{1}\right)}^{u_{1}}\right) h_{1}^{\prime} \cdots h_{r_{1}-1}^{\prime} C_{h\left(u_{j_{0}}\right)}^{0}\left(C_{1}^{0}-C_{h\left(u_{r_{1}+1}\right)}^{u_{r_{1}+1}}\right) \\
& \cdots h_{r_{k}-1}^{\prime} C_{h\left(u_{j_{0}}\right)}^{0}\left(C_{1}^{0}-C_{h\left(u_{r_{k}+1}\right)}^{u_{r_{k}+1}}\right) \cdots h_{3 k}^{\prime}=0 .
\end{aligned}
$$

Now since $R$ is $S$-Armendariz, by Lemma 2.8 ,

$$
\begin{aligned}
& \left(C_{1}^{0}-C_{h\left(u_{1}\right)}^{u_{1}}\right)(0) h_{1}^{\prime}\left(u_{1}\right) \cdots h_{r_{1}-1}^{\prime}\left(u_{r_{1}-1}\right) C_{h\left(u_{j_{0}}\right)}^{0}(0)\left(C_{1}^{0}-C_{h\left(u_{r_{1}+1}\right)}^{u_{r_{1}+1}}\right)(0) \cdots \\
& h_{r_{k}-1}^{\prime}\left(u_{r_{k}-1}\right) C_{h\left(u_{j_{0}}\right)}^{0}(0)\left(C_{1}^{0}-C_{h\left(u_{r_{k}+1}\right)}^{u_{r_{k}}}\right)(0) \cdots h_{3 k}^{\prime}\left(u_{3 k}\right) \\
= & h\left(u_{1}\right) h\left(u_{2}\right) \cdots h\left(u_{3 k}\right)=0 .
\end{aligned}
$$

Therefore we have prove that for each

$$
\left(u_{1}, u_{2}, \ldots, u_{3 k}\right) \in X_{w}(\underbrace{h, \ldots, h}_{3 k}), \quad h\left(u_{1}\right) h\left(u_{2}\right) \cdots h\left(u_{3 k}\right)=0 .
$$

Hence for any $w \in S, h^{3 k}(w)=0$, and so $h^{3 k}=(f g)^{3 k}=0$. Then by Lemma 2.8 , we obtain $(f(s) g(2 s))^{3 k}=(a+b c)^{3 k}=0$. Hence $a+b c \in \operatorname{nil}(R)$ is proved. Then by analogy with the proof of R. Antoine [2], Lemma 3.1(d), we can show that $a-b \in \operatorname{nil}(R)$. Hence $R$ is an $N I$ ring. Therefore by Proposition $2.2, R$ is nil generalized power serieswise Armendariz.

The following example shows that there exists a nil generalized power serieswise Armendariz ring which is not $S$-Armendariz. Hence a nil generalized power serieswise Armendariz ring is not a trivial extension of an $S$-Armendariz ring.

Example 2.10. Let $(S, \leq)$ be a strictly totally ordered monoid satisfying the condition that $0 \leq s$ for every $s \in S$, and $R$ a nil generalize power serieswiae Armendariz ring. Then by Proposition 2.5, $S_{4}(R)$ is nil generalized power serieswise Armendariz. Let $A=\left(\begin{array}{llll}0 & 1 & 0 & 0 \\ 0 & 0 & 0 & 0 \\ 0 & 0 & 0 & 0 \\ 0 & 0 & 0 & 0\end{array}\right), B=\left(\begin{array}{cccc}0 & 1 & -1 & 0 \\ 0 & 0 & 0 & 0 \\ 0 & 0 & 0 & 0 \\ 0 & 0 & 0 & 0\end{array}\right)$, $C=\left(\begin{array}{llll}0 & 0 & 0 & 0 \\ 0 & 0 & 0 & 0 \\ 0 & 0 & 0 & 1 \\ 0 & 0 & 0 & 0\end{array}\right), D=\left(\begin{array}{llll}0 & 0 & 0 & 0 \\ 0 & 0 & 0 & 1 \\ 0 & 0 & 0 & 1 \\ 0 & 0 & 0 & 0\end{array}\right)$ be four elements in $S_{4}(R)$. Let $0 \neq s \in S$. Define $f, g \in\left[\left[\left(S_{4}(R)\right)^{S, \leq}\right]\right]$ via

$$
f(x)=\left\{\begin{array}{l}
A \text { if } x=0, \\
B \text { if } x=s, \\
0 \text { otherwise, }
\end{array} \quad \text { and } \quad g(x)=\left\{\begin{array}{l}
C \text { if } x=0, \\
D \text { if } x=s, \\
0 \text { otherwise }
\end{array}\right.\right.
$$

Then $f g=0$, but $f(0) g(s) \neq 0$. So $S_{4}(R)$ is not $S$-Armendariz. 
Proposition 2.11. Let $S$ be a torsion-free and cancellative monoid, $\leq$ a strict order on $S$, and $I$ an nil ideal of $R$ (that is, $I \subseteq \operatorname{nil}(R)$ ). Then $R$ is nil generalized power serieswise Armendariz if and only if $R / I$ is nil generalized power serieswise Armendariz.

Proof. By analogy with the proof of [7, Proposition 5], we complete the proof.

It was shown in [8, Proposition 3.10] that if $I$ is a reduced ideal of $R$ such that $R / I$ is power serieswise Armendariz then $R$ is power serieswise Armendariz. Here we have the following result:

Proposition 2.12. Let $S$ be a torsion-free and cancellative monoid, $\leq$ a strict order on $S$, and $I$ an ideal of $R$. If $I$ is semicommutative and $R / I$ is nil generalized power serieswise Armendariz, then $R$ is nil generalized power serieswise Armendariz.

Proof. Let $f, g \in\left[\left[R^{S, \leq}\right]\right]$ be such that $f g \in\left[\left[\operatorname{nil}(R)^{S, \leq}\right]\right]$. By Ribenbiom [15], there exists a compatible strict total order $\leq^{\prime}$ on $S$, which is finer than $\leq$ (that is, for all $s, t \in S, s \leq t$ implies $\left.s \leq^{\prime} t\right)$. We will use transfinite induction on the strictly totally ordered set $\left(S, \leq^{\prime}\right)$ to show that $f(u) g(v) \in \operatorname{nil}(R)$ for any $u, v \in S$. Let $s$ and $t$ denote the minimum elements of $\operatorname{supp}(f)$ and $\operatorname{supp}(g)$ in the $\leq^{\prime}$ order, respectively. If $u \in \operatorname{supp}(f)$ and $v \in \operatorname{supp}(g)$ are such that $u+v=s+t$, then $s \leq^{\prime} u$ and $t \leq^{\prime} v$. If $s<^{\prime} u$, then $s+t<^{\prime} u+v=s+t$, a contradiction. Thus $u=s$. Similarly, $v=t$. Hence

$$
(f g)(s+t)=\sum_{(u, v) \in X_{s+t}(f, g)} f(u) g(v)=f(s) g(t) \in \operatorname{nil}(R)
$$

because $f g \in\left[\left[\operatorname{nil}(R)^{S, \leq}\right]\right]$.

Now suppose that $w \in S$ is such that for any $u, v \in S$ with $u+v<^{\prime} w$, $f(u) g(v) \in \operatorname{nil}(R)$. We will show that $f(u) g(v) \in \operatorname{nil}(R)$ for any $u, v \in S$ with $u+v=w$. We write

$$
X_{w}(f, g)=\{(u, v) \in S \times S \mid u+v=w, u \in \operatorname{supp}(f), v \in \operatorname{supp}(g)\}
$$

as $\left\{\left(u_{i}, v_{i}\right) \mid i=1,2, \ldots, n\right\}$ such that

$$
u_{1}<^{\prime} u_{2}<^{\prime} \cdots<^{\prime} u_{n} \text {. }
$$

Since $S$ is cancellative, $u_{1}=u_{2}$ and $u_{1}+v_{1}=u_{2}+v_{2}=w$ imply $v_{1}=v_{2}$. Since $\leq^{\prime}$ is a strict order, $u_{1}<^{\prime} u_{2}$ and $u_{1}+v_{1}=u_{2}+v_{2}=w$ imply $v_{2}<^{\prime} v_{1}$. Thus we have

$$
v_{n}<^{\prime} v_{n-1}<^{\prime} \cdots<^{\prime} v_{2}<^{\prime} v_{1}
$$

Now,

$$
(f g)(w)=\sum_{(u, v) \in X_{w}(f, g)} f(u) g(v)=\sum_{i=1}^{n} f\left(u_{i}\right) g\left(v_{i}\right) \in \operatorname{nil}(R) .
$$

For any $i \geq 2, u_{1}+v_{i}<^{\prime} u_{i}+v_{i}=w$, and thus, by induction hypothesis, we have $f\left(u_{1}\right) g\left(v_{i}\right) \in \operatorname{nil}(R)$. 
On the other hand, if we denote by $\bar{f}, \bar{g}$ the corresponding generalized power series of $f$ and $g$ in $\left[\left[(R / I)^{S, \leq}\right]\right], \bar{f} \bar{g} \in\left[\left[(\operatorname{nil}(R / I))^{S, \leq}\right]\right]$. There exists $n_{i j} \in \mathbb{N}$ such that $\left(f\left(u_{i}\right) g\left(v_{j}\right)\right)^{n_{i j}} \in I$ since $R / I$ is nil generalized power serieswise Armendariz. Then by analogy with the proof of Z. K. Liu [11], Theorem 3.6, we can show that $f(u) g(v) \in \operatorname{nil}(R)$ for any $u, v \in S$ with $u+v=w$. Hence by transfinite induction, $f(u) g(v) \in \operatorname{nil}(R)$ for any $u, v \in S$. Therefore $R$ is nil generalized power serieswise Armendariz.

Proposition 2.13. Let $S$ be a torsion-free and cancellative monoid, $\leq$ a strict order on $S$, and $R$ a nil generalized power serieswise Armendariz ring. If $f_{1}, f_{2}, \ldots, f_{n} \in\left[\left[R^{S, \leq}\right]\right]$ are such that $f_{1} f_{2} \cdots f_{n} \in\left[\left[\operatorname{nil}(R)^{S, \leq}\right]\right]$, then $f_{1}\left(u_{1}\right)$ $f_{2}\left(u_{2}\right) \cdots f_{n}\left(u_{n}\right) \in \operatorname{nil}(R)$ for all $u_{1}, u_{2}, \ldots, u_{n} \in S$.

Proof. Suppose $f_{1} f_{2} \cdots f_{n} \in\left[\left[\operatorname{nil}(R)^{S, \leq}\right]\right]$. Then from $f_{1}\left(f_{2} \cdots f_{n}\right) \in\left[\left[\operatorname{nil}(R)^{S, \leq}\right]\right]$, it follows that $f_{1}\left(u_{1}\right)\left(f_{2} \cdots f_{n}\right)(v) \in \operatorname{nil}(R)$ for all $u_{1}, v \in S$. Thus $\left(C_{f_{1}\left(u_{1}\right)}^{0} f_{2} \cdots\right.$ $\left.f_{n}\right)(v) \in \operatorname{nil}(R)$ for any $v \in S$, and so $C_{f_{1}\left(u_{1}\right)}^{0} f_{2} \cdots f_{n} \in\left[\left[\operatorname{nil}(R)^{S, \leq}\right]\right]$. Now from $\left(C_{f_{1}\left(u_{1}\right)}^{0} f_{2}\right)\left(f_{3} \cdots f_{n}\right) \in\left[\left[\operatorname{nil}(R)^{S, \leq}\right]\right]$, it follows that $\left(C_{f_{1}\left(u_{1}\right)}^{0} f_{2}\right)\left(u_{2}\right)\left(f_{3} \cdots\right.$ $\left.f_{n}\right)(w) \in \operatorname{nil}(R)$ for all $u_{2}, w \in S$. Since

$$
\left(C_{f_{1}\left(u_{1}\right)}^{0} f_{2}\right)\left(u_{2}\right)=f_{1}\left(u_{1}\right) f_{2}\left(u_{2}\right) \text { for any } u_{1}, u_{2} \in S,
$$

we have

$$
f_{1}\left(u_{1}\right) f_{2}\left(u_{2}\right)\left(f_{3} \cdots f_{n}\right)(w) \in \operatorname{nil}(R) \text { for all } u_{1}, u_{2}, w \in S
$$

Hence

$$
C_{\left(f_{1}\left(u_{1}\right) f_{2}\left(u_{2}\right)\right)}^{0} f_{3} \cdots f_{n} \in\left[\left[\operatorname{nil}(R)^{S, \leq}\right]\right] .
$$

Continuing this manner, we see that $f_{1}\left(u_{1}\right) f_{2}\left(u_{2}\right) \cdots f_{n}\left(u_{n}\right) \in \operatorname{nil}(R)$ for all $u_{1}, u_{2}, \ldots, u_{n} \in S$.

Corollary 2.14. Let $S$ be a torsion-free and cancellative monoid, $\leq$ a strict order on $S$. Then the following conditions are equivalent:

(1) If $f_{1}, f_{2}, \ldots, f_{n} \in\left[\left[R^{S, \leq}\right]\right]$ satisfy $f_{1} f_{2} \cdots f_{n} \in\left[\left[\operatorname{nil}(R)^{S, \leq}\right]\right]$, then $f_{1}\left(u_{1}\right)$ $f_{2}\left(u_{2}\right) \cdots f_{n}\left(u_{n}\right) \in \operatorname{nil}(R)$ for all $u_{1}, u_{2}, \ldots, u_{n} \in S$.

(2) $R$ is an NI ring.

Corollary 2.15. Let $S$ be a torsion-free and cancellative monoid, $\leq$ a strict order on $S$ and $R$ a nil generalized power serieswise Armendariz ring. Then $\operatorname{nil}\left(\left[\left[R^{S, \leq}\right]\right]\right) \subseteq\left[\left[\operatorname{nil}(R)^{S, \leq}\right]\right]$.

Proof. It follows from Proposition 2.13.

Recall that a ring $R$ is said to be have bounded index of nilpotency if there exists an integer $n \geq 1$ such that $x^{n}=0$ for each nilpotent element of $R$.

Corollary 2.16. Let $S$ be a torsion-free and cancellative monoid, $\leq$ a strict order on $S$ and $R$ a ring. If $\left[\left[\operatorname{nil}(R)^{S, \leq}\right]\right] \subseteq \operatorname{nil}\left(\left[\left[R^{S, \leq}\right]\right]\right)$, then $R$ has bounded index of nilpotency. 
Proof. Otherwise, for any positive integer $n$, there exists $a_{n} \in \operatorname{nil}(R)$ such that $a_{n}^{n} \neq 0$. Let $0 \neq s \in S$. Define $f \in\left[\left[R^{S, \leq}\right]\right]$ via

$$
f(x)= \begin{cases}a_{n} & \text { if } x=n ! s, \quad n=1,2, \ldots, \\ 0 & \text { otherwise. }\end{cases}
$$

Then $f \in\left[\left[\operatorname{nil}(R)^{S, \leq}\right]\right]$, and $\operatorname{supp}(f)=\{s, 2 ! s, 3 ! s, \ldots, n ! s, \ldots\}$. Since $\left[\left[\operatorname{nil}(R)^{S, \leq}\right]\right]$ $\subseteq \operatorname{nil}\left(\left[\left[R^{S, \leq}\right]\right]\right)$, there exists $k \geq 2$ such that $f^{k}=0$. For $n>k$, we have

$$
\begin{aligned}
f^{k}(k(n ! s)) & =\sum_{\left(u_{1}, u_{2}, \ldots, u_{k}\right) \in X_{(k n ! s)}(\underbrace{f, \ldots, f}_{k})} f\left(u_{1}\right) f\left(u_{2}\right) \cdots f\left(u_{k}\right) \\
& =f(n ! s) f(n ! s) \cdots f(n ! s)=a_{n}^{k} \neq 0
\end{aligned}
$$

which is impossible.

Proposition 2.17. Let $S$ be a torsion-free and cancellative monoid, $\leq$ a strict order on $S$, and $R$ a nil generalized power serieswise Armendariz ring. If $\operatorname{nil}(R)$ is nilpotent, then $\left[\left[\operatorname{nil}(R)^{S, \leq]}\right]=\operatorname{nil}\left(\left[\left[R^{S, \leq}\right]\right]\right)\right.$.

Proof. By Corollary 2.15, we have $\left[\left[\operatorname{nil}(R)^{S, \leq]]} \supseteq \operatorname{nil}\left(\left[\left[R^{S, \leq}\right]\right]\right)\right.\right.$. So if suffices to show that $\left[\left[\operatorname{nil}(R)^{S, \leq}\right]\right] \subseteq \operatorname{nil}\left(\left[\left[R^{S,} \leq\right]\right]\right)$. Assume that $f \in\left[\left[\operatorname{nil}(R)^{S, \leq}\right]\right]$. Since $\operatorname{nil}(R)$ is nilpotent, there exists some positive integer $k$ such that $(\operatorname{nil}(R))^{k}=0$. So for any $s \in S$,

$$
\left.f^{k}(s)=\sum_{\left(u_{1}, u_{2}, \ldots, u_{k}\right) \in X_{s}}(\underbrace{f, \ldots, f}_{k})\right)
$$

Thus $f^{k}=0$, and so $f \in \operatorname{nil}\left(\left[\left[R^{S, \leq}\right]\right]\right)$. Hence $\left[\left[\operatorname{nil}(R)^{S, \leq}\right]\right] \subseteq \operatorname{nil}\left(\left[\left[R^{S, \leq}\right]\right]\right)$. Therefore $\left[\left[\operatorname{nil}(R)^{S, \leq}\right]\right]=\operatorname{nil}\left(\left[\left[R^{S, \leq}\right]\right]\right)$.

Corollary 2.18. Let $S$ be a torsion-free and cancellative monoid, $\leq$ a strict order on $S$, and $R$ a nil generalized power serieswise Armendariz right noetherian ring. Then $\left[\left[\operatorname{nil}(R)^{S, \leq}\right]\right]=\operatorname{nil}\left(\left[\left[R^{S, \leq}\right]\right]\right)$.

Proof. Since $R$ is nil generalized power serieswise Armendariz, by Proposition $2.2, R$ is an $N I$ ring. Then by the well known Levitzki's Theorem [9], $\operatorname{nil}(R)$ is nilpotent. Hence the result follows from Proposition 2.17.

N. K. Kim et al. have shown in [8, Proposition 3.1] that a ring $R$ is power serieswise Armendariz if and only if $R[x]$ is power serieswise Armendariz. For nil power serieswise Armendariz rings, S. Hizem have shown in [7, Corollary 7] that if $R$ is a semicommutative ring, then $R[x]$ is a nil power serieswise Armendariz ring. As to nil generalized power serieswise Armendariz rings, we have the following:

Proposition 2.19. Let $S$ be a torsion-free and cancellative monoid, $\leq$ a strict order on $S$, and $R$ a nil generalized power serieswise Armendariz ring. If nil $(R)$ is nilpotent, then $\left[\left[R^{S,} \leq\right]\right]$ is nil generalized power serieswise Armendariz for any torsion-free cancellative monoid $T$ and any strict order $\leq_{T}$ on $T$. 
Proof. Since $R$ is nil generalized power serieswise Armendariz, by Proposition $2.2, R$ is an $N I$ ring. Since $\operatorname{nil}(R)$ is nilpotent, by Proposition 2.17, we have $\left[\left[\operatorname{nil}(R)^{S, \leq}\right]\right]=\operatorname{nil}\left(\left[\left[R^{S, \leq}\right]\right]\right)$, and so $\left[\left[R^{S, \leq}\right]\right]$ is an $N I$ ring. Then by Proposition $2.2,\left[\left[R^{S, \leq}\right]\right]$ is nil generalized power serieswise Armendariz for any torsion-free cancellative monoid $T$ and any strict order $\leq_{T}$ on $T$.

Corollary 2.20. Let $S$ be a torsion-free and cancellative monoid, $\leq$ a strict order on $S$, and $R$ a nil generalized power serieswise Armendariz right noetherian ring. Then $\left[\left[R^{S, \leq}\right]\right]$ is nil generalized power serieswise Armendariz for any torsion-free cancellative monoid $T$ and any strict order $\leq_{T}$ on $T$.

\section{Nilpotent property of nil generalized power serieswise Armendariz rings}

Let $U$ and $V$ be two sets of $R$. We use $U: V$ to represent the set $\{x \in R \mid$ $V x \subseteq U\}$. Then for any $U \subseteq R$, we have

$$
\begin{aligned}
\operatorname{nil}(R): U & =\{x \in R \mid U x \subseteq \operatorname{nil}(R)\} \\
& =\{x \in R \mid x U \subseteq \operatorname{nil}(R)\} .
\end{aligned}
$$

If $\operatorname{nil}(R)$ is an ideal, then $\operatorname{nil}(R): U$ is an ideal of $R$ for any subset $U \subseteq R$, and $\left[\left[\operatorname{nil}(R)^{S, \leq]}\right]: V\right.$ is also an ideal of $\left[\left[R^{S, \leq}\right]\right]$ for any subset $V$ of $\left[\left[R^{S, \leq}\right]\right]$. Given a generalized power series $f \in\left[\left[R^{S, \leq}\right]\right]$, let $C_{f}$ denote the set $\{f(s) \mid s \in \operatorname{supp}(f)\}$, and for a subset $V \subseteq\left[\left[R^{S, \leq}\right]\right]$, let $C_{V}$ denote the set $\bigcup_{f \in V} C_{f}$.

Given a ring $R$, we define

$$
N A n n_{R}\left(2^{R}\right)=\{\operatorname{nil}(R): U \mid U \subseteq R\}
$$

and

$$
N A n n_{\left[\left[R^{S, \leq]}\right]\right.}\left(2^{\left[\left[R^{S, \leq}\right]\right]}\right)=\left\{\left[\left[\operatorname{nil}(R)^{S, \leq}\right]\right]: V \mid V \subseteq\left[\left[R^{S, \leq}\right]\right]\right\}
$$

Proposition 3.1. Let $S$ be a torsion-free and cancellative monoid, $\leq$ a strict order on $S$, and $R$ a nil generalized power serieswise Armendariz ring. Then

$$
\phi: N A n n_{R}\left(2^{R}\right) \longrightarrow N A n n_{\left[\left[R^{S, \leq}\right]\right]}\left(2^{\left[\left[R^{S, \leq}\right]\right]}\right)
$$

defined by $\phi(I)=\left[\left[I^{S, \leq}\right]\right]$ for every $I \in N A n n_{R}\left(2^{R}\right)$ is bijective.

Proof. We first prove that $\left[\left[(\operatorname{nil}(R): U)^{S, \leq]}\right]=\left[\left[\operatorname{nil}(R)^{S, \leq}\right]\right]: U\right.$ for any subset $U \subseteq R$. Suppose that $f \in\left[\left[(\operatorname{nil}(R): U)^{S, \leq}\right]\right]$. Then for any $s \in S$, $f(s) \in \operatorname{nil}(R): U$, and so for any $r \in U, f(s) r=\left(f C_{r}^{0}\right)(s) \in \operatorname{nil}(R)$. Hence $f C_{r}^{0} \in\left[\left[\operatorname{nil}(R)^{S, \leq]}\right]\right.$, and so $f \in\left[\left[\operatorname{nil}(R)^{S, \leq]}\right]: U\right.$. Thus $\left[\left[(\operatorname{nil}(R): U)^{S, \leq]}\right] \subseteq\right.$ $\left[\left[\operatorname{nil}(R)^{S, \leq]}\right]: U\right.$. Now we claim that $\left[\left[\operatorname{nil}(R)^{S, \leq}\right]\right]: U \subseteq\left[\left[(\operatorname{nil}(R): U)^{S, \leq}\right]\right]$. For any generalized power series $f \in\left[\left[\operatorname{nil}(R)^{S, \leq]}\right]: U\right.$, and any $r \in U$, we have $f r=f C_{r}^{0} \in\left[\left[\operatorname{nil}(R)^{S, \leq}\right]\right]$. Then for any $s \in S,\left(f C_{r}^{0}\right)(s)=f(s) r \in \operatorname{nil}(R)$, and so for any $s \in S, f(s) \in \operatorname{nil}(R): U$. Hence $f \in\left[\left[(\operatorname{nil}(R): U)^{S, \leq]]}\right.\right.$. Thus $\left[\left[\operatorname{nil}(R)^{S, \leq]}\right]: U \subseteq\left[\left[(\operatorname{nil}(R): U)^{S, \leq]}\right]\right.\right.$. Hence $\left[\left[\operatorname{nil}(R)^{S, \leq}\right]\right]: U=[[(\operatorname{nil}(R):$ $U)^{S, \leq]]}$ is proved, and so $\phi$ is well defined. 
We next claim that $\phi$ is injective. Let

$$
\begin{aligned}
& I_{1}=\operatorname{nil}(R): U_{1} \in N A n n_{R}\left(2^{R}\right), \\
& I_{2}=\operatorname{nil}(R): U_{2} \in N A n n_{R}\left(2^{R}\right),
\end{aligned}
$$

and

$$
\operatorname{nil}(R): U_{1} \neq \operatorname{nil}(R): U_{2} .
$$

Then $\left[\left[\left(\operatorname{nil}(R): U_{1}\right)^{S, \leq}\right]\right] \neq\left[\left[\left(\operatorname{nil}(R): U_{2}\right)^{S, \leq}\right]\right]$ is clear. Hence $\phi\left(I_{1}\right) \neq \phi\left(I_{2}\right)$. So $\phi$ is injective.

Finally, we show that $\phi$ is surjective. Let $\left[\left[\operatorname{nil}(R)^{S, \leq]]}: V \in N A n_{\left[\left[R^{S,} \leq\right]\right]}\right.\right.$ $\left(2^{\left[\left[R^{S,}\right]\right]}\right)$, where $V \subseteq\left[\left[R^{S, \leq}\right]\right]$. We wish to claim that

$$
\left[\left[\operatorname{nil}(R)^{S, \leq}\right]\right]: V=\left[\left[\left(\operatorname{nil}(R): C_{V}\right)^{S, \leq}\right]\right]=\phi\left(\operatorname{nil}(R): C_{V}\right)
$$

Let $f \in\left[\left[\operatorname{nil}(R)^{S, \leq}\right]\right]: V$. Then $f g \in\left[\left[\operatorname{nil}(R)^{S, \leq}\right]\right]$ for any $g \in V$. Since $R$ is nil generalized power serieswise Armendariz, $f(u) g(v) \in \operatorname{nil}(R)$ for any $u, v \in S$. Thus for any $u \in S, f(u) C_{V} \subseteq \operatorname{nil}(R)$ and so for any $u \in S$, $f(u) \in \operatorname{nil}(R): C_{V}$. Then $f \in\left[\left[\left(\operatorname{nil}(R): C_{V}\right)^{S, \leq]}\right]\right.$ and so $\left[\left[\operatorname{nil}(R)^{S, \leq}\right]\right]: V \subseteq$ $\left[\left[\left(\operatorname{nil}(R): C_{V}\right)^{S, \leq]}\right]\right.$. Conversely, assume that $f \in\left[\left[\left(\operatorname{nil}(R): C_{V}\right)^{S, \leq}\right]\right]$. Then for any $u \in S, f(u) C_{V} \subseteq \operatorname{nil}(R)$. Hence for any $g \in V$, it is easy to see that $f g \in\left[\left[\operatorname{nil}(R)^{S, \leq}\right]\right]$. So $f \in\left[\left[\operatorname{nil}(R)^{S, \leq}\right]\right]: V$. Hence $\left[\left[\left(\operatorname{nil}(R): C_{V}\right)^{S, \leq}\right]\right] \subseteq$ $\left[\left[\operatorname{nil}(R)^{S, \leq]]}: V\right.\right.$. Thus

$$
\left[\left[\operatorname{nil}(R)^{S, \leq}\right]\right]: V=\left[\left[\left(\operatorname{nil}(R): C_{V}\right)^{S, \leq}\right]\right]=\phi\left(\operatorname{nil}(R): C_{V}\right) .
$$

Hence $\phi$ is surjective. Therefore $\phi$ is a bijection.

Corollary 3.2. Let $N$ Ann $n_{R[[x]]}\left(2^{R[[x]]}\right)=\{\operatorname{nil}(R)[[x]]: V \mid V \subseteq R[[x]]\}$, and let $N \operatorname{Ann}_{R\left[\left[x_{1}, \ldots, x_{n}\right]\right]}\left(2^{R\left[\left[x_{1}, \ldots, x_{n}\right]\right]}\right)=\left\{\operatorname{nil}(R)\left[\left[x_{1}, \ldots, x_{n}\right]\right]: V \mid V \subseteq R\left[\left[x_{1}, \ldots, x_{n}\right]\right]\right\}$. Then we have the following results.

(1) If $R$ is nil power serieswise Armendariz, then

$$
\phi: N A n n_{R}\left(2^{R}\right) \longrightarrow N A n n_{R[[x]]}\left(2^{R[[x]]}\right)
$$

defined by $\phi(I)=I[[x]]$ for every $I \in N A n n_{R}\left(2^{R}\right)$ is bijective.

(2) If $R$ is $n$ nil power serieswise Armendariz, then

$$
\phi: N A n n_{R}\left(2^{R}\right) \longrightarrow N A n n_{R\left[\left[x_{1}, \ldots, x_{n}\right]\right]}\left(2^{R\left[\left[x_{1}, \ldots, x_{n}\right]\right]}\right)
$$

defined by $\phi(I)=I\left[\left[x_{1}, \ldots, x_{n}\right]\right]$ for every $I \in N A n_{R}\left(2^{R}\right)$ is bijective.

Proof. By Proposition 3.1, we complete the proof.

Proposition 3.3. Let $S$ be a torsion-free and cancellative monoid, $\leq$ a strict order on $S$, and $R$ a nil generalized power serieswise Armendariz ring. If for each nonempty subset $X \nsubseteq \operatorname{nil}(R), \operatorname{nil}(R): X$ is generated as a right ideal by a nilpotent element, then for each nonempty subset $U \subseteq\left[\left[R^{S, \leq}\right]\right]$ with $U \nsubseteq\left[\left[\operatorname{nil}(R)^{S, \leq}\right]\right],\left[\left[\operatorname{nil}(R)^{S, \leq}\right]\right]: U$ is generated as a right ideal by a nilpotent element. 
Proof. Let $U$ be a nonempty subset of $\left[\left[R^{S, \leq}\right]\right]$ with $U \nsubseteq\left[\left[\operatorname{nil}(R)^{S, \leq]}\right]\right.$. Suppose that $f \in\left[\left[\operatorname{nil}(R)^{S, \leq]}\right]: U\right.$. Then $f g \in\left[\left[\operatorname{nil}(R)^{S, \leq]}\right]\right.$ for each $g \in U$. Since $R$ is nil generalized power serieswise Armendariz, $f(u) g(v) \in \operatorname{nil}(R)$ for each $u$, $v \in S$. Hence for any $u \in S, f(u) \in \operatorname{nil}(R): C_{U}$. If $C_{U} \subseteq \operatorname{nil}(R)$, then $U \subseteq\left[\left[\operatorname{nil}(R)^{S, \leq]}\right]\right.$, a contradiction. Hence there exists $p \in \operatorname{nil}(R)$ such that $\operatorname{nil}(R): C_{U}=p R$. Now we show that $\left[\left[\operatorname{nil}(R)^{S, \leq]}\right]: U=C_{p}^{0}\left[\left[R^{S, \leq}\right]\right]\right.$. Note that for any $u \in S, f(u) \in \operatorname{nil}(R): C_{U}=p R$. Thus for any $u \in S$, there exists $r_{u} \in R$ such that $f(u)=p r_{u}$. Define $h \in\left[\left[R^{S, \leq}\right]\right]$ via

$$
h(x)=\left\{\begin{array}{cl}
r_{u} & \text { if } \quad x=u \in \operatorname{supp}(f) \quad \text { and } \quad f(u)=p r_{u} \\
0 & \text { otherwise }
\end{array}\right.
$$

Then $f=C_{p}^{0} h \in C_{p}^{0}\left[\left[R^{S, \leq}\right]\right]$. Hence $\left[\left[\operatorname{nil}(R)^{S, \leq}\right]\right]: U \subseteq C_{p}^{0}\left[\left[R^{S, \leq}\right]\right]$.

On the other hand, for each $g \in\left[\left[R^{S,} \leq\right]\right], f \in U$ and each $s \in S$,

$$
\left(C_{p}^{0} g f\right)(s)=\sum_{(v, u) \in X_{s}(g, f)} p g(v) f(u) .
$$

Since $R$ is nil generalized power serieswise Armendariz, by Proposition 2.2, $R$ is an $N I$ ring. Then it is easy to see that $\left(C_{p}^{0} g f\right)(s) \in \operatorname{nil}(R)$ for each $s \in S$. Hence $C_{p}^{0} g f \in\left[\left[\operatorname{nil}(R)^{S, \leq}\right]\right]$. Thus $C_{p}^{0}\left[\left[R^{S, \leq]} \subseteq\left[\left[\operatorname{nil}(R)^{S, \leq}\right]\right]: U\right.\right.$. Therefore $\left[\left[\operatorname{nil}(R)^{S, \leq]]}: U=C_{p}^{0}\left[\left[R^{S, \leq]}\right]\right.\right.\right.$ where $C_{p}^{0} \in \operatorname{nil}\left(\left[\left[R^{S, \leq}\right]\right]\right)$.

Proposition 3.4. Let $(S, \leq)$ be a torsion-free cancellative strictly ordered monoid satisfying the condition that $s \geq 0$ for all $s \in S$ and $R$ a nil generalized power serieswise Armendariz ring. Then the following conditions are equivalent:

(1) For each nonempty subset $X \nsubseteq \operatorname{nil}(R), \operatorname{nil}(R): X$ is generated as a right ideal by a nilpotent element.

(2) For each nonempty subset $U \subseteq\left[\left[R^{S, \leq}\right]\right]$ with $U \nsubseteq\left[\left[\operatorname{nil}(R)^{S, \leq}\right]\right],\left[\left[\operatorname{nil}(R)^{S, \leq}\right]\right]$ : $U$ is generated as a right ideal by a nilpotent element.

Proof. (1) $\Longrightarrow(2)$ is immediate from Proposition 3.3.

$(2) \Longrightarrow(1)$ Let $X$ be a nonempty subset of $R$ with $X \nsubseteq \operatorname{nil}(R)$. Then $X \nsubseteq\left[\left[\operatorname{nil}(R)^{S, \leq}\right]\right]$. Then $\left[\left[\operatorname{nil}(R)^{S, \leq]]}: X=f\left[\left[R^{S, \leq}\right]\right]\right.\right.$ where $f$ is a nilpotent element of $\left[\left[R^{S, \leq}\right]\right]$. Since $R$ is nil generalized power serieswise Armendariz, by Corollary 2.15, $f \in\left[\left[\operatorname{nil}(R)^{S, \leq}\right]\right]$. Hence for any $s \in S, f(s) \in \operatorname{nil}(R)$. In particular, $f(0) \in \operatorname{nil}(R)$. Now we show that $\operatorname{nil}(R): X=f(0) R$. Since $R$ is nil generalized power serieswise Armendariz, by Proposition 2.2, $R$ is an $N I$ ring. Then it is easy to see that $f(0) R \subseteq n i l(R): X$. So it suffices to show that $\operatorname{nil}(R): X \subseteq f(0) R$. If $m \in \operatorname{nil}(R): X$, then $C_{m}^{0} \in\left[\left[\operatorname{nil}(R)^{S, \leq]}\right]\right.$ : $X=f\left[\left[R^{S, \leq}\right]\right]$. There exists $g \in\left[\left[R^{S, \leq}\right]\right]$ such that $C_{m}^{0}=f g$. Since $(S, \leq)$ is torsion-free cancellative strictly ordered monoid satisfying the condition that $s \geq 0$ for all $s \in S$, we have $C_{m}^{0}(0)=m=(f g)(0)=f(0) g(0) \in f(0) \cdot R$, and so $\operatorname{nil}(R): X \subseteq f(0) \cdot R$. Hence $\operatorname{nil}(R): X=f(0) \cdot R$ with $f(0) \in \operatorname{nil}(R)$. Therefore, $\operatorname{nil}(R): X$ is generated as a right ideal by a nilpotent element. 
Proposition 3.5. Let $S$ be a torsion-free and cancellative monoid, $\leq$ a strict order on $S$, and $R$ a nil generalized power serieswise Armendariz ring. If for each $p \notin \operatorname{nil}(R), \operatorname{nil}(R): p$ is generated as a right ideal by a nilpotent element, then for each $f \notin\left[\left[\operatorname{nil}(R)^{S, \leq}\right]\right],\left[\left[\operatorname{nil}(R)^{S, \leq]}\right]: f\right.$ is generated as a right ideal by a nilpotent element.

Proof. Let $f \notin\left[\left[\operatorname{nil}(R)^{S, \leq}\right]\right]$. Suppose that $g \in\left[\left[\operatorname{nil}(R)^{S, \leq}\right]\right]: f$. Then $f g \in\left[\left[\operatorname{nil}(R)^{S, \leq}\right]\right]$. Since $R$ is nil generalized power serieswise Armendariz, $f(u) g(v) \in \operatorname{nil}(R)$ for all $u, v \in S$. Thus $g(v) \in \operatorname{nil}(R): f(u)$ for all $u, v \in S$. If for all $u \in S, f(u) \in \operatorname{nil}(R)$, then $f \in\left[\left[\operatorname{nil}(R)^{S, \leq}\right]\right]$, a contradiction. Thus there exists $u \in S$ such that $f(u) \notin \operatorname{nil}(R)$, and so there exists $q \in \operatorname{nil}(R)$ such that $\operatorname{nil}(R): f(u)=q R$. Now we show that $\left[\left[\operatorname{nil}(R)^{S, \leq}\right]\right]: f=C_{q}^{0}\left[\left[R^{S, \leq}\right]\right]$. Note that for any $v \in S, g(v) \in \operatorname{nil}(R): f(u)=q R$. So for any $v \in S$, there exists $r_{v} \in R$ such that $g(v)=q r_{v}$. Define $h \in\left[\left[R^{S, \leq}\right]\right]$ via

$$
h(x)=\left\{\begin{array}{cl}
r_{v} & \text { if } x=v \in \operatorname{supp}(g) \text { and } g(v)=q r_{v}, \\
0 & \text { otherwise. }
\end{array}\right.
$$

Then $g=C_{q}^{0} h \in C_{q}^{0}\left[\left[R^{S, \leq}\right]\right]$, and so $\left[\left[\operatorname{nil}(R)^{S, \leq}\right]\right]: f \subseteq C_{q}^{0}\left[\left[R^{S, \leq}\right]\right]$.

On the other hand, for each $h \in\left[\left[R^{S,} \leq\right]\right]$ and each $s \in S$,

$$
\left(C_{q}^{0} h f\right)(s)=\sum_{(u, v) \in X_{s}(h, f)} q h(u) f(v) .
$$

Since $R$ is nil generalized power serieswise Armendariz, by Proposition 2.2, $R$ is an NI ring. Then it is easy to see that $\left(C_{q}^{0} h f\right)(s) \in \operatorname{nil}(R)$ for all $s \in S$. Hence $C_{q}^{0} h f \in\left[\left[\operatorname{nil}(R)^{S, \leq}\right]\right]$. Thus $C_{q}^{0}\left[\left[R^{S, \leq}\right]\right] \subseteq\left[\left[\operatorname{nil}(R)^{S, \leq}\right]\right]: f$. Therefore $\left[\left[\operatorname{nil}(R)^{S, \leq]]}: f=C_{q}^{0}\left[\left[R^{S, \leq}\right]\right]\right.\right.$ where $C_{q}^{0} \in \operatorname{nil}\left(\left[\left[R^{S, \leq}\right]\right]\right)$.

Proposition 3.6. Let $S$ be a torsion-free cancellative strictly ordered monoid satisfying the condition that $s \geq 0$ for all $s \in S$ and $R$ a nil generalized power serieswise Armendariz ring. Then the following conditions are equivalent:

(1) For each $p \notin \operatorname{nil}(R), \operatorname{nil}(R): p$ is generated as a right ideal by a nilpotent element.

(2) For each $f \notin\left[\left[\operatorname{nil}(R)^{S, \leq}\right]\right],\left[\left[\operatorname{nil}(R)^{S, \leq}\right]\right]: f$ is generated as a right ideal by a nilpotent element.

Proof. It is similar to the proof as given in Proposition 3.4,

Corollary 3.7. If $R$ is a nil power serieswise Armendariz ring, then the following conditions are equivalent:

(1) For each nonempty subset $X \nsubseteq \operatorname{nil}(R), \operatorname{nil}(R): X$ is generated as a right ideal by a nilpotent element.

(2) For each nonempty subset $U \subseteq R[[x]]$ with $U \subseteq \operatorname{nil}(R)[[x]], \operatorname{nil}(R)[[x]: U$ is generated as a right ideal by a nilpotent element.

Corollary 3.8. If $R$ is an $n$ nil power serieswise Armendariz ring, then the following conditions are equivalent: 
(1) For each nonempty subset $X \nsubseteq \operatorname{nil}(R), \operatorname{nil}(R): X$ is generated as a right ideal by a nilpotent element.

(2) For each nonempty subset $U \subseteq R\left[\left[x_{1}, \ldots, x_{n}\right]\right]$ with $U \nsubseteq \operatorname{nil}(R)\left[\left[x_{1}, \ldots, x_{n}\right]\right]$, $\operatorname{nil}(R)\left[\left[x_{1}, \ldots, x_{n}\right]: U\right.$ is generated as a right ideal by a nilpotent element.

Corollary 3.9. If $R$ is a nil power serieswise Armendariz ring, then the following conditions are equivalent:

(1) For each $p \notin n i l(R), \operatorname{nil}(R): p$ is generated as a right ideal by a nilpotent element.

(2) For each $f \notin n i l(R)[[x]], \operatorname{nil}(R)[[x]]: f$ is generated as a right ideal by a nilpotent element.

Corollary 3.10. If $R$ is an $n$ nil power serieswise Armendariz ring, then the following conditions are equivalent:

(1) For each $p \notin n i l(R), \operatorname{nil}(R): p$ is generated as a right ideal by a nilpotent element.

(2) For each $f \notin \operatorname{nil}(R)\left[\left[x_{1}, \ldots, x_{n}\right]\right], \operatorname{nil}(R)\left[\left[x_{1}, \ldots, x_{n}\right]\right]: f$ is generated as a right ideal by a nilpotent element.

For any subset $X$ of a ring $R, r_{R}(X)=\{a \in R \mid X a=0\}$ denotes the right annihilator of $X$ in $R$. Faith [6] called a ring $R$ right zip provided that if the right annihilator $r_{R}(X)$ of a subset $X$ of $R$ is zero, then there exists a finite subset $Y \subseteq X$ such that $r_{R}(Y)=0$. Beachy and Blair [4] showed that if $R$ is a commutative zip ring, then the polynomial ring $R[x]$ over $R$ is a zip ring. As a generalization of zip rings, in [13] L. Ouyang introduced the notion of weak zip rings. A ring $R$ is a weak zip ring provided that for any subset $X$ of $R$, if $\operatorname{nil}(R): X \subseteq \operatorname{nil}(R)$, then there exists a finite subset $Y \subseteq X$ such that $\operatorname{nil}(R): Y \subseteq \operatorname{nil}(R)$. L. Ouyang showed that if $R$ is a semicommutative ring, then $R$ is weak zip if and only if $R[x]$ is weak zip. In the following we investigate the weak zip property of the generalized power series ring $\left[\left[R^{S, \leq}\right]\right]$ under the condition that $R$ is nil generalized power serieswise Armendariz.

Lemma 3.11. Let $(S, \leq)$ be a cancellative torsion-free strictly ordered monoid and $R$ a nil generalized power serieswise Armendariz ring. Then the following conditions are equivalent:

(1) $R$ is a weak zip ring.

(2) For each subset $U \subseteq\left[\left[R^{S, \leq}\right]\right]$, if $\left[\left[\operatorname{nil}(R)^{S, \leq}\right]\right]: U \subseteq\left[\left[\operatorname{nil}(R)^{S, \leq}\right]\right]$, then

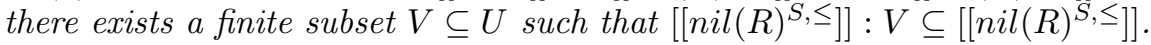

Proof. $(1) \Longrightarrow(2)$ Let $U$ be a subset of $\left[\left[R^{S, \leq}\right]\right]$ such that $\left[\left[\operatorname{nil}(R)^{S, \leq]]}: U \subseteq\right.\right.$ $\left[\left[\operatorname{nil}(R)^{S, \leq]}\right]\right.$. We first show that $\operatorname{nil}(R): C_{U} \subseteq \operatorname{nil}(R)$. Suppose that $a \in$ $\operatorname{nil}(R): C_{U}$. Then for any $f \in U$ and any $s \in S,\left(f C_{a}^{0}\right)(s)=f(s) a \in \operatorname{nil}(R)$. Thus for any $f \in U, f C_{a}^{0} \in\left[\left[\operatorname{nil}(R)^{S, \leq}\right]\right]$, and so $C_{a}^{0} \in\left[\left[\operatorname{nil}(R)^{S, \leq}\right]\right]: U \subseteq$ $\left[\left[\operatorname{nil}(R)^{S, \leq]}\right]\right.$. Hence $a=C_{a}^{0}(0) \in \operatorname{nil}(R)$ and so $\operatorname{nil}(R): C_{U} \subseteq \operatorname{nil}(R)$ is proved. Since $R$ is a weak zip ring, there exists a finite subset $Y \subseteq C_{U}$ such that $\operatorname{nil}(R): C_{U} \subseteq \operatorname{nil}(R)$. Without loss of generality, we may assume that $Y=\left\{u_{1}, u_{2}, \ldots, u_{n}\right\} \subseteq C_{U}$. For each $u_{i} \in Y$, there exists some $f_{u_{i}} \in U$ such 
that $f_{u_{i}}\left(s_{i}\right)=u_{i}$ for some $s_{i} \in S$. Let $V$ be a minimal subset of $U$ such that $f_{u_{i}} \in V$ for each $u_{i} \in Y, 1 \leq i \leq n$. Then $V$ is a finite subset of $U$ and $Y \subseteq C_{V}$. Now we show that $\left[\left[\operatorname{nil}(R)^{S, \leq]]}: V \subseteq\left[\left[\operatorname{nil}(R)^{S, \leq}\right]\right]\right.\right.$. Suppose $h \in\left[\left[\operatorname{nil}(R)^{S, \leq]]}\right.\right.$ : $V$. Then $g h \in\left[\left[\operatorname{nil}(R)^{S, \leq]}\right]\right.$ for every $g \in V$. Then $g(u) h(v) \in \operatorname{nil}(R)$ for each $u, v \in S$ since $R$ is nil generalized power serieswise Armendariz, and so for any $v \in S, h(v) \in \operatorname{nil}(R): C_{V} \subseteq \operatorname{nil}(R): Y \subseteq \operatorname{nil}(R)$. Hence $h \in\left[\left[\operatorname{nil}(R)^{S, \leq}\right]\right]$. Therefore $\left[\left[\operatorname{nil}(R)^{S, \leq}\right]\right]: V \subseteq\left[\left[\operatorname{nil}(R)^{S, \leq}\right]\right]$, as desired.

$(2) \Longrightarrow(1)$ Assume that $\operatorname{nil}(R): X \subseteq \operatorname{nil}(R)$ where $X$ is a subset of $R$. We first show that $\left[\left[\operatorname{nil}(R)^{S, \leq}\right]\right]: X \subseteq\left[\left[\operatorname{nil}(R)^{S, \leq}\right]\right]$. Let $f \in\left[\left[\operatorname{nil}(R)^{S, \leq}\right]\right]: X$. Then $x f=C_{x}^{0} f \in\left[\left[\operatorname{nil}(R)^{S, \leq}\right]\right]$ for each $x \in X$. Since $R$ is nil generalized power serieswise Armendariz, $x f(s) \in \operatorname{nil}(R)$ for each $s \in S$ and $x \in X$. Thus for each $s \in S, f(s) \in \operatorname{nil}(R): X \subseteq \operatorname{nil}(R)$. This implies that $f \in\left[\left[\operatorname{nil}(R)^{S, \leq}\right]\right]$ and so $\left[\left[\operatorname{nil}(R)^{S, \leq]]}: X \subseteq\left[\left[\operatorname{nil}(R)^{S, \leq}\right]\right]\right.\right.$. Then we can find a finite subset $Y \subseteq X$ such that $\left[\left[\operatorname{nil}(R)^{S, \leq]]}: Y \subseteq\left[\left[\operatorname{nil}(R)^{S, \leq}\right]\right]\right.\right.$. Now we show that $\operatorname{nil}(R): Y \subseteq \operatorname{nil}(R)$. If $a \in \operatorname{nil}(R): Y$, then $C_{a}^{0} \in\left[\left[\operatorname{nil}(R)^{S, \leq}\right]\right]: Y \subseteq\left[\left[\operatorname{nil}(R)^{S, \leq}\right]\right]$, and so $a \in \operatorname{nil}(R)$. Hence $\operatorname{nil}(R): Y \subseteq \operatorname{nil}(R)$. Therefore $R$ is a weak zip ring.

Proposition 3.12. Let $(S, \leq)$ be a cancellative torsion-free strictly ordered monoid and $R$ a nil generalized power serieswise Armendariz ring with nil $(R)$ nilpotent. Then the following conditions are equivalent:

(1) $R$ is a weak zip ring.

(2) $\left[\left[R^{S, \leq}\right]\right]$ is a weak zip ring.

Proof. By Proposition 2.17 and Proposition 3.9, we complete the proof.

Corollary 3.13. We have the following results.

(1) If $R$ is a nil power serieswise Armendariz with nil $(R)$ nilpotent. Then $R$ is a weak zip ring if and only if the power series ring $R[[x]]$ is weak zip.

(2) If $R$ is an $n$ nil power serieswise Armendariz with nil $(R)$ nilpotent. Then $R$ is a weak zip ring if and only if the power series ring $R\left[\left[x_{1}, \ldots, x_{n}\right]\right]$ in $n$ indeterminates is weak zip.

Corollary 3.14. We have the following results.

(1) If $R$ is a nil power serieswise Armendariz right noetherian ring. Then $R$ is a weak zip ring if and only if the power series ring $R[[x]]$ is weak zip.

(2) If $R$ is an $n$ nil power serieswise Armendariz right noetherian ring. Then $R$ is a weak zip ring if and only if the power series ring $R\left[\left[x_{1}, \ldots, x_{n}\right]\right]$ in $n$ indeterminates is weak zip.

Acknowledgement. This research is supported by the National Natural Science Foundation of China (10771058, 11071062), Natural Science Foundation of Hunan Province (10jj3065) and Scientific Research Fundation of Hunan Provincial Education Department (10A033).

\section{References}

[1] D. D. Anderson and V. Camillo, Armendariz rings and Gaussian rings, Comm. Algebra 26 (1998), no. 7, 2265-2272. 
[2] R. Antoine, Nilpotent elements and Armendariz rings, J. Algebra 319 (2008), no. 8, 3128-3140.

[3] E. P. Armendariz, A note on extensions of Baer and p.p.-rings, J. Austral. Math. Soc. 18 (1974), 470-473.

[4] J. A. Beachy and W. D. Blair, Rings whose faithful left ideals are cofaithful, Pacific J. Math. 58 (1975), no. 1, 1-13.

[5] H. Cartan and E. Eilenberg, Homological Algebra, Princeton Landmarks in Mathematics, originally published in 1956, Princeton: Princeton University Press, 1956.

[6] C. Faith, Rings with zero intersection property on annihilators: zip rings, Publ. Mat. 33 (1989), no. 2, 329-332.

[7] S. Hizem, A note on nil power serieswise Armendariz rings, Rendiconti del Circolo Mathematico di Palermo 59 (2010), no. 1, 87-99.

[8] N. K. Kim, K. H. Lee, and Y. Lee, Power series rings satisfying a zero divisor property, Comm. Algebra 34 (2006), no. 6, 2205-2218.

[9] T. Y. Lam, A First Course in Noncommutative Rings, Graduate Texts in Mathematics, Springer-Verlag, Berlin, 1991

[10] Z. K. Liu, Special properties of rings of generalized power series, Comm. Algebra 32 (2004), no. 8, 3215-3226.

[11] _ On weak Armendariz rings, Comm. Algebra 34 (2006), no. 7, 2607-2616.

[12] L. Ouyang, Ore extensions of weak zip rings, Glasg. Math. J. 51 (2009), no. 3, 525-537.

[13] M. B. Rege and S. Chhawchharia, Armendariz rings, Proc. Japan Acad. Ser. A Math. Sci. 73 (1997), no. 1, 14-17.

[14] P. Ribenboim, Rings of generalized power series: Nilpotent elements, Abh. Math. Sem. Univ. Hamburg 61 (1991), 15-33.

[15] _ Noetherian rings of generalized power series, J. Pure Appl. Algebra 79 (1992), no. 3, 293-312.

[16] _ Semisimple rings and von Neumann regular rings of generalized power series, J. Algebra 198 (1997), no. 2, 327-338.

OUYANG LUNQUN

Department of Mathematics

Hunan University of Science and Technology

Xiangtan, Hunan 411201, P. R. China

E-mail address: ouyanglqtxy@163.com

LIU JINWANG

Department of Mathematics and Applied Mathematics

HuAihua University

Huainua, 418000, P. R. China

E-mail address: jwliu64@yahoo.com.cn 